\title{
The ICV Sign as a Marker of Increased Cerebral Blood Transit Time
}

\author{
Bijoy K. Menon, Helin Daniel Bai, Jayesh Modi, Andrew M. Demchuk \\ Mark Hudon, Mayank Goyal, Timothy W.J. Watson
}

\begin{abstract}
Objective/Background: We describe the internal cerebral vein (ICV) sign, which is a hypo-opacification of the ICV on computed tomogram angiography (CTA) as a new marker of increased cerebral blood transit-time in ipsilateral internal carotid artery occlusions (ICAO). Methods: A retrospective analysis of 153 patients with acute unilateral M1 middle cerebral artery (MCA) occlusions \pm ICAOs was performed. The degree of contrast opacification of the ICV on the ipsilesional side was compared to that of the unaffected side. Results: Of 153 patients in our study, 135 had M1 MCA occlusions \pm intra-cranial ICAO (M1 \pm iICAO) and 18 had isolated extracranial ICAO (eICAO). In the patients with proximal M1 \pm iICAO, 57/65 (87.1\%) showed the ICV sign. Of the 8 patients without the ICV sign in this group, 6 had prominent lenticulostriate arteries arising from the non-occluded M1 segment, 1 had a recurrent artery of Huebner, and 1 had filling of distal ICA/M1 segment through prominent Circle of Willis collaterals. For the 70 patients with isolated distal M1 $\mathrm{iICAO}, 7 / 70(10 \%)$ showed the ICV sign, with all 7 showing occluded lenticulostriate arteries. Of the patients with eICAO, 8/18 showed the ICV sign, all 8 with the ICV sign had poor Circle of Willis collaterals. Conclusions: The ICV sign correlates well with presence of proximal M1 $\mathrm{iICAO}$ in patients with either occluded lenticulostriate arteries or poor Circle of Willis collaterals. In patients with eICAO, the sign correlates with reduced Circle of Willis collaterals and may be a marker of increased ipsilateral cerebral blood transit time.
\end{abstract}

RÉSUMÉ: Le signe de la veine cérébrale interne comme marqueur du temps de transit du flux sanguin cérébral. Contexte et objectif : Nous décrivons le signe de la veine cérébrale interne (VCI), visualisé comme une hypo-opacification de la veine cérébrale interne (VIC) à l'angiographie par tomodensitométrie (ACT), comme un nouveau marqueur de l'augmentation du temps de transit du flux sanguin cérébral dans les occlusions de la carotide interne ipsilatérale (OCII). Méthode : Nous avons effectué une analyse rétrospective des dossiers de 153 patients atteints d'une occlusion aiguë unilatérale du segment M1 de l'artère cérébrale moyenne (ACM) avec ou sans OCII. Le degré d'opacification de la VCI du même côté que la lésion a été comparé à celui du côté sans lésion. Résultats : Parmi ces 153 patients, 135 avaient une occlusion du segment M1 de l'ACM avec ou sans OCII intracrânienne (M1 \pm OCIIi) et 18 avaient une OCII extracrânienne isolée (OCIIe). Chez les patients atteints d'une occlusion du segment M1 proximal \pm OCIIi, 57/65 (87,1\%) présentaient le signe de la VCI et 6 des 8 patients sans signe de la VCI avaient des artères lenticulostriées proéminentes prenant naissance d'un segment M1 non occlus, 1 avait une artère récurrente de Huebner et 1 avait un remplissage de la carotide interne/du segment distal M1 par l'intermédiaire de collatérales proéminentes de l'hexagone de Willis. Parmi les 70 patients présentant une occlusion du segment M1 \pm OCIIi 7 patients $(10 \%)$ présentaient le signe de la VCI et tous les 7 avaient des artères lenticulostriée occluses. Parmi les 18 patients atteints de OCIIe, 8 présentaient le signes de la VCI et tous les 8 avaient une mauvaise circulation collatérale au niveau de l'hexagone de Willis. Conclusions : Nous avons observé une bonne corrélation entre le signe de la VCI et la présence d'occlusion de la M1 proximale \pm OCIIi chez les patients porteurs d'artères lenticulostriées occluses ou d'une mauvaise circulation collatérale au niveau de l'hexagone de Willis. Chez les patients atteints d'OCIIe, il existe une bonne corrélation entre le signe de la VCI et une circulation collatérale diminuée au niveau de l'hexagone de Willis, ce qui pourrait être un marqueur d'un temps de transit du flux sanguin cérébral ipsilatéral augmenté.

Can J Neurol Sci. 2013; 40: 187-191

The assessment of cerebral blood flow in the clinical setting is technologically challenging. Although a number of imaging modalities exist, issues of availability, operational complexity, and relative invasiveness have made them non-ideal for investigating cerebral perfusion. For example, positron emission tomography, which is the gold standard, is still primarily used as a research tool $^{1,2}$. Computed tomogram/magnetic resonance (CT/MR) perfusion techniques, shown to be relatively accurate, are limited by methodological assumptions, post-acquisition processing techniques, and inconsistent delay correction algorithms ${ }^{3,4}$. Digital subtraction angiography, although capable of providing high-resolution images, is relatively invasive and not optimal for measuring cerebral perfusion ${ }^{5}$.
Computed tomogram angiography (CTA) is a widely used technique in ischemic stroke cases for investigating thrombus site, arterial anatomy, and collateral status ${ }^{6}$. Assessing the venous contrast phase in CTAs can provide valuable insight into

From the Department of Clinical Neurosciences (BKM, AMD, MG, TWJW), Department of Radiology (JM, HDB, MH, MG), University of Calgary, Calgary, Alberta, Canada.

Received May 10, 2012. Final Revisions Submitted September 17, 2012. Correspondence to: Bijoy K. Menon, Department of Clinical Neurosciences, University of Calgary, 1403, 29th Street NW, Calgary, Alberta, T2N 2T9, Canada. Email: Bijoy.Menon@albertahealthservices.ca. 
cerebral tissue perfusion through using arteriovenous transit times $^{7,8}$. In this article, we shall focus on this imaging tool and its potential utility in detecting increased blood transit time in ischemic stroke patients via the visualization of unilateral internal cerebral vein (ICV) hypo-opacification.

The ICVs are located near the inter-ventricular foramen ${ }^{9}$. These two veins drain the deep cerebral hemispheric structures, which include: midline structures like the fornix, septum pellucidum, corpus callosum, basal ganglia, thalamus, medial temporal lobe, and hippocampus ${ }^{10}$. The ICVs drain the thalamostriate and anterior septal veins, which receive their arterial supply from predominantly four arteries: the lateral lenticulostriate arteries arising from the M1 middle cerebral artery (MCA) segment, the medial lenticulostriate arteries arising from the A1 segment of the anterior cerebral artery (ACA), the recurrent artery of Heubner arising from either the A2 or A1 segment of the ACA, and the anterior choroidal artery $^{11}$. Thus, patients presenting with unilateral ICA and proximal M1 occlusions will likely have poor blood flow to the anterior choroidal and lateral lenticulostriate arteries, which would lead to poor perfusion of deep cerebral structures and ultimately cause poor/delayed filling of the ipsilateral ICV.

We hypothesize that in ischemic stroke patients, the ICV sign i.e. the hypo-opacification of a unilateral ICV on CTA is a marker of increased ipsilateral deep cerebral hemisphere blood transit time.

\section{Materials AND Methods}

From a retrospective study of 1240 subjects presenting to our centre between 2003-09 with either acute ischemic strokes or transient ischemic attacks undergoing CTA, 153 patients with intra-cranial M1 MCA \pm internal carotid artery occlusions $(\mathrm{M} 1 \pm \mathrm{iICAO})$ or with extra-cranial internal carotid artery occlusions (eICAO) were selected to be studied. The study was approved by the local institutional review board.

Standard non-helical, non-contrast CT (NCCT) scans were performed with multi-slice scanners (GE Medical Systems or Siemens) using $120 \mathrm{kV}, 170 \mathrm{mAs}$ and $5 \mathrm{~mm}$ thick slices. Scan coverage from skull base to vertex used continuous axial slices parallel to the orbitomeatal line. After the NCCTs, CTAs were performed using helical scans. Acquisitions were obtained after a single bolus (90-120 ml) of intravenous-nonionic contrast was injected into an ante-cubital vein at $3-5 \mathrm{ml} / \mathrm{sec}$. Imaging was manually initiated at the appearance of contrast in the ascending aorta. The minimum scan coverage was from arch to vertex using $0.6 \mathrm{~mm}$ to $1.25 \mathrm{~mm}$ thick slices. After imaging, a CT technologist immediately created multi-planar volumereconstructed images with 2.5 to $4.0 \mathrm{~mm}$ thick slices in axial, sagittal, and coronal planes. OsiriX software (Pixmeo, Geneva, Switzerland) was then used to generate 2D multi-planar reconstructions from the CTA in three planes using $40 \mathrm{~mm}$ thick slabs $^{12}$.

Two authors (BM and JM), reviewed and scored by consensus the baseline CT angiograms for the presence of the internal cerebral vein while being blinded to patient clinical information and follow up scans. The ICV sign was considered positive if contrast density at the most proximal $2 \mathrm{~cm}$ of the internal cerebral vein where it joins the basal vein of Rosenthal to form the vein of Galen was less than the contralateral side. Similar contrastation but reduced size was not considered a positive sign as this appearance could be due to anatomical variations including absent ICV in the distalmost portions.

The data is described using standard descriptive statistics. Patients with $\mathrm{M} 1 \pm \mathrm{iICAO}$ and those with eICAO were analyzed separately. We used Fisher's exact test for categorical data. Interrater reliability for the ICV sign was assessed on 30 patients using a kappa's test. A $p$ value of $<$ or $=0.05$ was considered statistically significant. Stata 12 was used to perform data analysis.

\section{RESULTS}

Of the 153 patients included in the study ( 74 male), 135 were

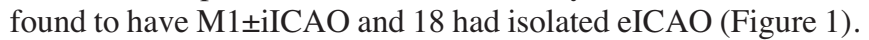
The median National Institute of Health Stroke Scale score was 16, median stroke onset time to CTA was 164 minutes with $>75 \%$ of patients being scanned at $<360$ minutes.

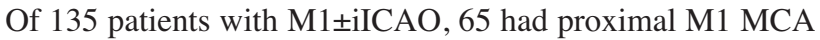
occlusions and 70 had isolated distal M1 MCA occlusions. Of the 65 patients in this group with proximal M1 MCA occlusions, $57 / 65(87.7 \%)$ showed the ICV sign, when compared to $7 / 70$

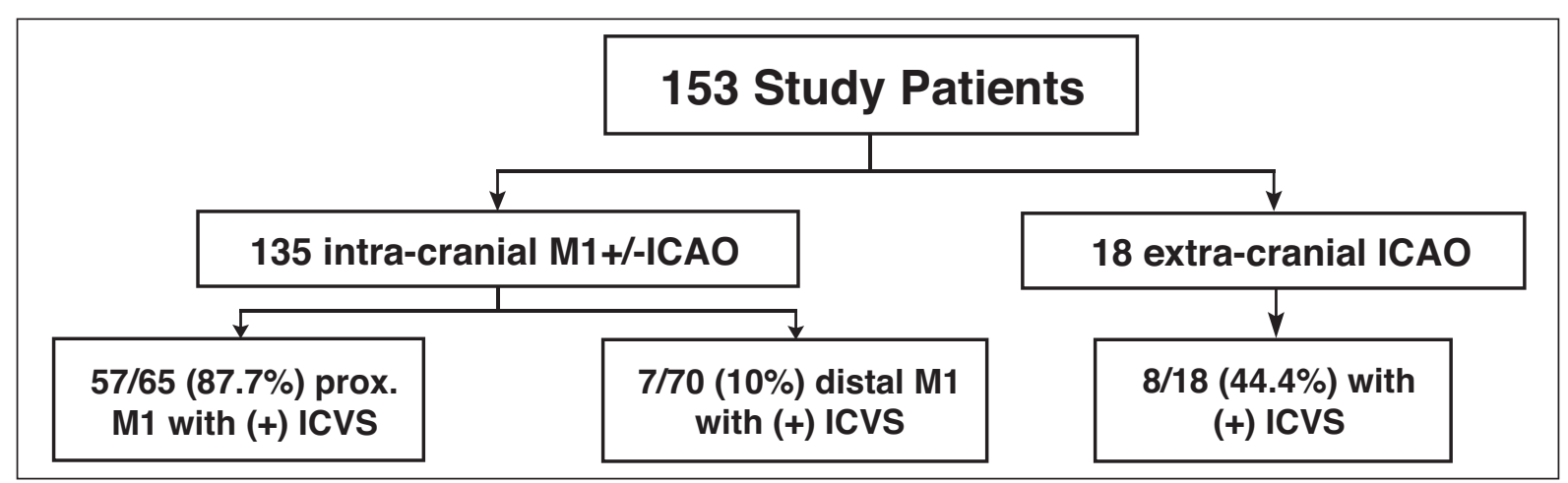

Figure 1: A flowchart of patients included in the study and their results with respect to positive internal cerebral vein (ICVS) sign. $M 1$ middle cerebral artery occlusions \pm intra-cranial internal carotid artery occlusions $(M 1 \pm i I C A O)$. prox=proximal; ICVS=internal cerebral vein sign; ICAO=internal carotid artery occlusion 


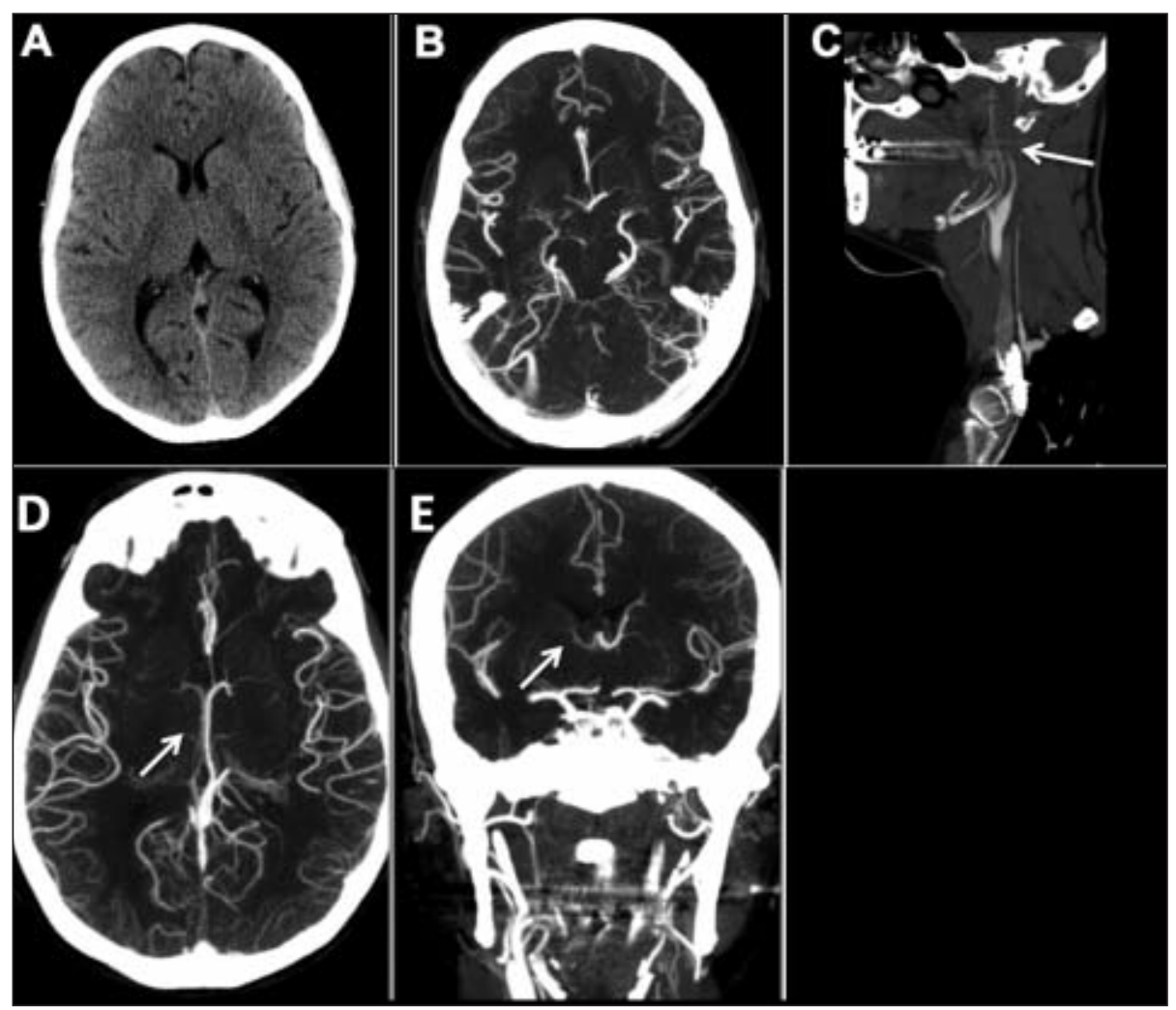

Figure 2: Imaging of a 35 year-old patient with acute left-sided weakness $12 \mathrm{hrs}$ after onset showing (a) normal NCCT scan (b) CTA source image with normal leptomeningeal collaterals in the right MCA territory, (c) CTA neck showing total occlusion of the right ICA. CTA MIP images shows hypo-opacification of right internal cerebral vein, arrows in (d) axial and (e) coronal reformatted MIP images

(10\%) with distal M1 MCA occlusions with a positive ICV sign (Figure 1). This difference was statistically significant $(\mathrm{p}<0.001)$.

Of the eight patients with proximal M1 MCA occlusions that didn't show an ICV sign, six had prominent lenticulostriate arteries arising from the non-occluded M1 segment, one had a recurrent artery of Heubner with perforating branches supplying the basal ganglia, and one had filling of the distal ICA segment from prominent anterior Circle of Willis collaterals (anterior communication artery and bilateral anterior cerebral artery A1 segments). Of the 7/70 patients who showed the ICV sign in the distal M1 MCA occlusion cohort, none had visible lenticulostriate arteries on coronal CTA.

In the 18 patients with isolated eICAO, 8/18 (44.4\%) showed a positive ICV sign (Figure 1). All eight patients with the ICV sign had poor anterior Circle of Willis collaterals (hypoplastic or absent anterior communicating artery or A1 ACA segments) and all ten patients without the sign demonstrated good anterior Circle of Willis collaterals.

Inter-rater reliability for the sign was excellent. (kappa $=0.92$, standard deviation $=0.18, \mathrm{p}<0.001$ )

\section{Discussion}

In patients with internal carotid artery occlusions, the occurrence of ischemic strokes has been strongly correlated with impaired cerebral hemodynamics ${ }^{13}$. The evaluation of cerebral hemodynamics, however, can be technologically challenging in many clinical settings using the available imaging tools ${ }^{14}$. Currently, most diagnostic centers use either conventional cerebral angiography or perfusion imaging (CT or MR) to estimate cerebral vascular sufficiency. For reasons of practicality, we propose an alterative technique to assess cerebral perfusion using venous hypo-opacification on CTA. Our proposed technique could be a useful screening tool due to widespread availability and use of CTA. However, one should note that this method is limited in its ability to only assess deep cerebral hemispheric structures that are supplied by the internal carotid and M1 MCA segments of the anterior circulation. Thus, this sign is an indicator of tissue blood flow transit time prolongation and may not necessarily correlate with tissue dysfunction.

The following is a clinical example of how the ICV sign helped us assess cerebral blood transit time in a patient suffering from acute ischemic stroke.

A 35-year-old female patient presented to us after a nine hour history of headache and collapse from left sided weakness. On examination, she had a right gaze preference, left partial hemianopia and left hemiparesis with ipsilateral facial paresis. Her National Institute of Health Stroke Scale at presentation was 12 and fluctuated from 8 to 12 over the over the next 1.5 hours. On imaging, NCCT brain was normal (Figure 2a). CTA head and neck showed patent intracranial arteries and good leptomeningeal collaterals on the ipsilesional hemisphere (Figure 2b, 2c) along with a near total occlusion of the right 

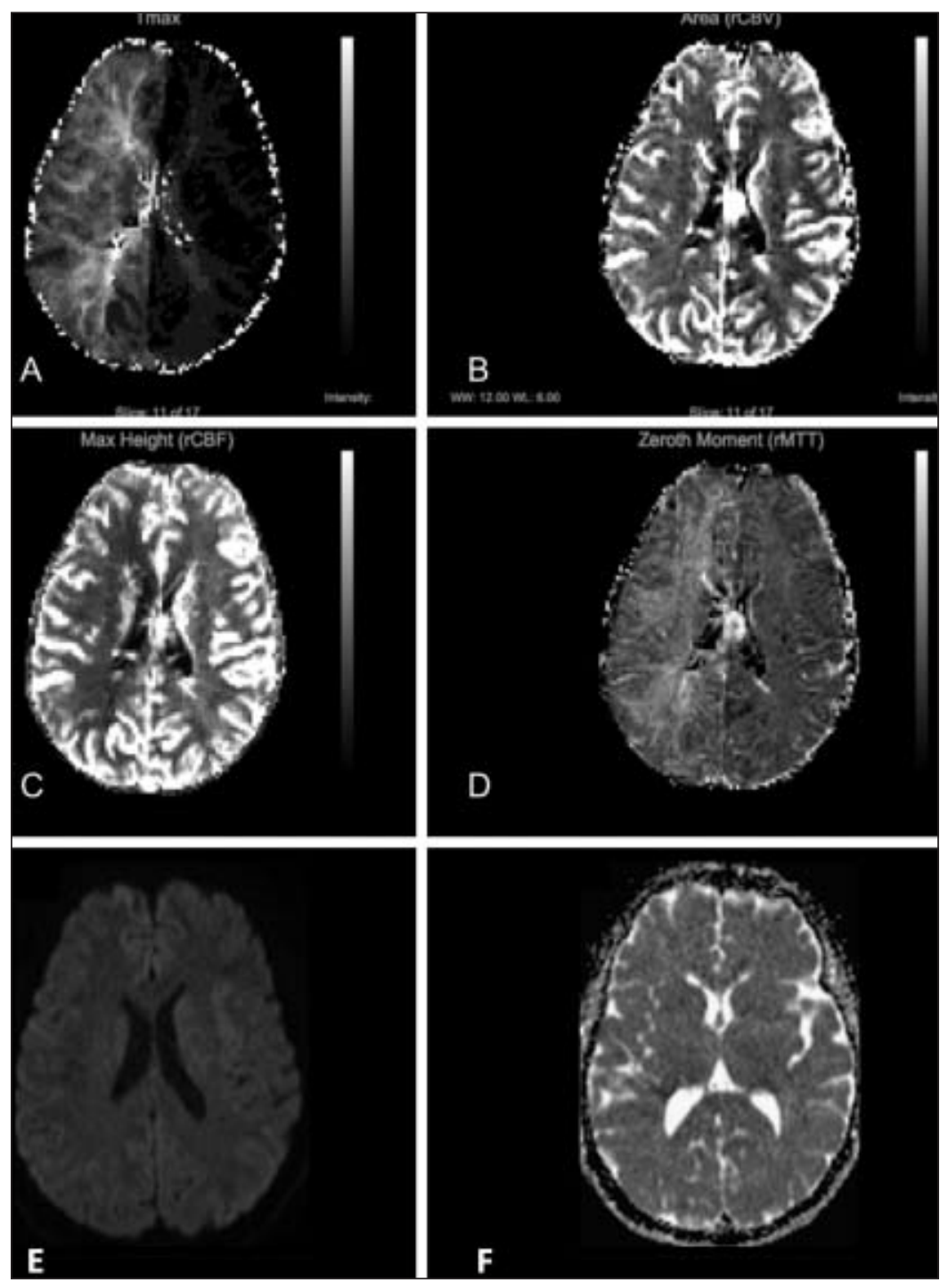

Figure 3: MRI perfusion study showing: (a) prolonged Tmax in right anterior circulation, (b) slightly increased blood volume via $C B V$ map, (c) CBF mapping showing slightly decreased blood flow in right anterior circulation, (d) and a prolonged MTT. An MRI diffusion study shows (e) normal DWI and $(f)$ normal $A D C$ maps. cervical ICA (Figure 2d, 2e). The right ICV was significantly hypo-opacificed when compared to the contralateral side (Figure 2 ). A MR perfusion study showed prolonged mean transit time, an increased cerebral blood volume, and decreased cerebral blood flow in the anterior circulation (Figure 3a, 3b, 3c, 3d). Moreover, the right M1 MCA segment distal to the ICA occlusion showed significantly reduced velocity and pulsatility index on trans-cranial Doppler ultrasound (Figure 4a, 4b). The diffusion weighted MR image and apparent diffusion coefficient maps showed no ischemic lesions (Figure 3e, 3f). After these multi-modal imaging results, cervical ICA stenting was performed (14 hours after the onset of initial symptoms). Over the next two days, the patient recovered very quickly and was discharged home without any neurological deficits. A follow-up NCCT brain was normal. In this patient, the ICV sign corresponded to signs of hypoperfusion on multimodal imaging and possibly to the clinical status of the patient, suggesting a potential utility as an immediate screening tool for increased blood transit time.

A majority of the patients with proximal M1+iICAO $(87.7 \%)$ in our study showed the ICV sign, suggesting that the sign is a good marker of delayed blood transit time in this group of patients. In eight patients in this group that did not show the sign, the presence of prominent anterior Circle of Willis collateral circulation and lenticulostriate arteries visualized on CTA supplying the ipsilesional ICV drainage territory further supports this hypothesis. Given that brain tissue supplied by the distal M1 segment of the MCA does not drain significantly into the ICV, we were not surprised to find that only $10 \%$ of patients with distal M1 occlusions showed the ICV sign (Figure 1). The ICV sign in this group with distal occlusions was only noticed when the distal M1 MCA occlusions blocked the lenticulostriate arteries. 


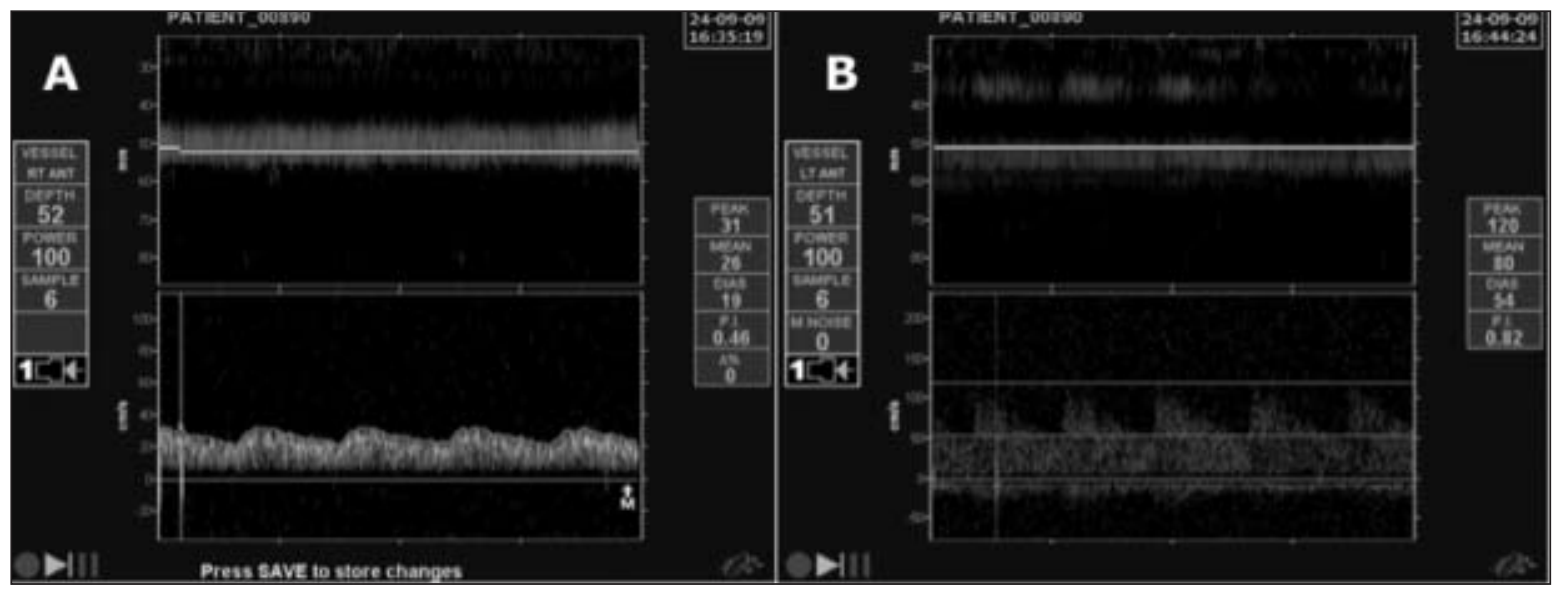

Figure 4: Transcranial doppler showing (a) significantly reduced velocity and pulsatility index in the right MI MCA segment distal to the ICA occlusion as compared to (b) normal left side.

Similar to the patients having M1 \pm iICAO, the $8 / 18$ patients with isolated eICAOs showing a positive ICV sign all had poor anterior Circle of Willis collaterals and the remaining ten patients without the sign all had good anterior Circle of Willis collaterals.

Our results support the hypothesis that ipsilateral ICV hypoopacification on CTA on the side of iICAOs or eICAOs is a marker of occluded lenticulostriate arteries or poor anterior Circle of Willis collaterals. In patients with isolated eICAOs with patent intracranial ICA and M1 MCA, the presence of a positive ICV sign reinforces the impression of insufficient anterior Circle of Willis collaterals. The ICV sign could be an early imaging mark of hypoperfusion in the deep hemispheric structures. It could suggest clot migration and hypoperfusion of the basal ganglia in patients with distal M1 MCA occlusions or fragmented distal territory MCA infarcts.

Our study is observational and has several limitations. A caveat of this study includes the need to recognize anatomical variations such as reduced ICV size and being able to differentiate it from true hypo-opacification. Lenticulostriate arteries are not always visualized on a conventional CTA. Furthermore, possible timing errors in CT bolus tracking may occur and, therefore, one must ensure that there is a consistent triggering protocol for contrast transit bilaterally into the venous phase. It is also important to recognize that this study is a preliminary descriptive analysis and that no correlations with respect to clinical status or outcomes have been drawn. Thus, the ICV sign must be interpreted only as a marker of deep increased blood transit time in cerebral tissue, as the sign's clinical relevance in each case depends upon clinician judgment. Further clarification on the sensitivity and specificity of the ICV sign as a marker of at risk brain tissue will require a prospective study with direct comparison to CT perfusion. A comparison study of this type will also be able to derive quantitative Hounsfield unit thresholds for hypo-opacification when assessing the sign. We also expect that this manuscript would generate interest amongst clinicians and radiologists to look at the venous aspects of a CTA in more detail as a marker of delayed transit time for blood flow.

\section{REFERENCES}

1. Yonas H, Good WF, Gur D, et al. Mapping cerebral blood flow by xenon-enhanced computed tomography: clinical experience. Radiology. 1984;152(2):435-42.

2. Neirinckx RD, Canning LR, Piper IM, et al. Technetium-99m d, 1-HM-PAO: a new radiopharmaceutical for SPECT imaging of regional cerebral blood perfusion. J Nucl Med. 1987;28(2): 191-202.

3. Kucharczyk J, Mintorovitch J, Asgari HS, Moseley M. Diffusion/perfusion MR imaging of acute cerebral ischemia. Magn Reson Med. 1991;19(2):311-5.

4. Hamberg LM, Hunter GJ, Halpern EF, Hoop B, Gazelle GS, Wolf GL. Quantitative high-resolution measurement of cerebrovascular physiology with slip-ring CT. AJNR Am J Neuroradiol. 1996;17(4):639-50.

5. Latchaw RE, Yonas H, Hunter GJ, et al. Guidelines and recommendations for perfusion imaging in cerebral ischemia: A scientific statement for healthcare professionals by the writing group on perfusion imaging, from the Council on Cardiovascular Radiology of the American Heart Association. Stroke. 2003;34 (4): 1084-104.

6. Grond M, Rudolf J, Schneweis S, et al. Feasibility of source images of computed tomographic angiography to detect the extent of ischemia in hyperacute stroke. Cerebrovasc Dis. 2002;13(4): 251-6.

7. Bhatia R, Bal SS, Shobha N, et al. CT angiographic source images predict outcome and final infarct volume better than noncontrast CT in proximal vascular occlusions. Stroke. 2011;42(6): 1575-80.

8. Dorn F, Liebig T, Muenzel D, et al. Order of CT stroke protocol (CTA before or after CTP): impact on image quality. Neuroradiology. 2011;54(2):105-12.

9. Taveras JM. Angiography in Neuroradiology. 3rd edition ed: Williams \& Wilkins; 1996.

10. Meder JF, Chiras J, Roland J, Guinet P, Bracard S, Bargy F. Venous territories of the brain. J Neuroradiol. 1994;21(2):118-33.

11. Kido DK, Baker RA. Normal Cerebral Vascular Anatomy. Boston: Little, Brown, and Company; 1983.

12. Rosset A, Spadola L, Ratib O. OsiriX: an open-source software for navigating in multidimensional DICOM images. J Digit Imaging. 2004;17(3):205-16.

13. Yonas H, Smith HA, Durham SR, Pentheny SL, Johnson DW. Increased stroke risk predicted by compromised cerebral blood flow reactivity. J Neurosurg. 1993;79(4):483-9.

14. Powers WJ. Cerebral hemodynamics in ischemic cerebrovascular disease. Ann Neurol. 1991;29(3):231-40. 